\title{
Los cielos y el fin de los tiempos. La astronomía en la obra teológica de Manuel Lacunza
}

\author{
Patricio Leyton A. \\ HPLEYTON@UC.CL \\ Zenobio Saldivia $M$. \\ DEPARTAMENTO DE HUMANIDADES \\ UNIVERSIDAD TECNOLÓGICA METROPOLITANA \\ zenobio@utem.cl
}

Resumen: Los trabajos sobre el jesuita chileno Manuel Lacunza han tratado aspectos biográficos, teológicos y editoriales, los cuales se han centrado en los temas escatológicos de La Venida del Mesías en Gloria y Majestad y en las ideas religiosas de su autor. Además, se suma a esto que los investigadores han abordado las diferentes ediciones de la obra y la recepción que tuvo esta tanto en Europa como en América, dejando un vacío en lo referente a las ideas científicas presentes en ella, las cuales están contenidas en el tercer tomo. Por lo tanto, en este artículo abordaremos las referencias astronómicas incluidas en el tratado teológico de Lacunza, la cual fue la ciencia que el jesuita chileno sintió afición durante su estadía en Chile como en Italia, desde la perspectiva de la historia de la ciencia. Para este cometido se analizará la ciencia y su vinculación con la religión en el pensamiento del teólogo jesuita proponiendo que no hubo una contradicción o conflicto en ambas vías.

Palabras clave: Lacunza, jesuitas, ciencia, religión y astronomía.

Abstract: The works on the Chilean Jesuit Manuel Lacunza have dealt with biographical, theological and editorial aspects, which have focused on the eschatological themes of The Coming of the Messiah in Glory and Majesty and in the religious ideas of its author. In addition, it is added to this that the researchers have approached the different editions of the work and the reception that it had both in Europe and in America, leaving a gap in relation to the scientific ideas present in it, which are contained in the Third volume Therefore, in this article we will address the astronomical references included in the theological treatise of Lacunza, which was the science that the Chilean Jesuit was fond of during his stay in Chile as in Italy, from the perspective of the history of science. For this purpose science and its connection with religion in the thought of the Jesuit theologian will be analyzed, proposing that there was no contradiction or conflict in both ways.

Key words: Lacunza, jesuits, science, religion and astronomy. 


\section{INTRODUCCIÓN}

Los trabajos investigativos sobre jesuitas destacados en América o sobre la Compañía de Jesús en general, y/o en especial sobre Chile, y en particular sobre su producción científica, están notoriamente muy difundidos; al respecto y en relación a este último país, baste recordar el libro de Enrich, o los textos y artículos de Hanisch, o el ensayo de González; o más recientemente, el texto de Saldivia y Caro, por ejemplo ${ }^{1}$. Pero no sucede lo mismo en relación al jesuita Manuel Lacunza, pues más allá de enfatizar en los aspectos biobibliográficos de su obra y en el estudio cuidadoso de las ediciones de La Venida del Mesías en Gloria y Majestad; sus comentaristas solo han abordado el análisis desde el punto de vista biográfico, teológico o editorial, así como también desde el plano de la difusión y recepción de la obra, o sobre la influencia de la ilustración católica y el contexto intelectual en que su autor escribió el texto ${ }^{2}$.

Sin embargo, no se ha realizado aún un análisis del escrito de Lacunza desde la perspectiva de la historia de la ciencia, o de algunos ejes epistémicos; ya que en la obra La Venida del Mesías en Gloria y Majestad hay varias referencias a temas científicos y en particular a la astronomía, y si bien el jesuita chileno no fue un científico formal, sino más bien un teólogo; a la historiografía de la ciencia le interesa en la actualidad, no solo estudiar a los científicos o a las instituciones en que estos se desarrollan, sino que a todos los agentes sociales y políticos que interactúen con el quehacer de la ciencia y con aquellos que inciden en las decisiones de la práctica científica, incluido a los legos y otros sujetos que tengan participación en la producción del conocimiento ${ }^{3}$.

1 De F. EnRICH, recuérdese su obra Historia de la Compañia de Jesús en Chile, Tomos I y II (Imprenta de Francisco Rosal, Barcelona: 1891). De W. Hanisch, Historia de la Compañia de Jesús en Chile (Editorial Francisco de Aguirre, Buenos Aires: 1974). De J. A. GonZÁlez, La Compañia de Jesús y la ciencia ilustrada. Juan Ignacio Molina y la Historia Natural y Civil de Chile (Ediciones Universitarias, Universidad Católica del Norte, Antofagasta: 1993). Y de Z. SAldivia - F. CAro, Cinco jesuitas relevantes en América y su aporte a las Ciencias (Editorial Universidad Tecnológica Metropolitana, Santiago: 2016).

2 Un recuento bibliográfico sobre estudios de Lacunza se puede encontrar en $\mathrm{O}$. ARCE, "Manuel Lacunza y La Venida del Mesías en gloria y majestad: bibliografía comentada", Revista Chilena de Literatura 73 (2008).

3 Ver J. Pimentel, “¿Qué es la historia cultural de la ciencia?”, Arbor 743 (2006). 
Por ello, en este artículo, se pretende cubrir dicha carencia historiográfica en relación a las aportaciones científicas del jesuita mencionado $\mathrm{y}$ de sus interacciones con la cultura del período.

El estudio del texto de Lacunza desde el marco de la historia de la ciencia, por lo tanto, nos permite entender el hecho de que algunas personas ajenas a la práctica científica contribuyen a difundir los saberes en ensayos que tienen una función totalmente distinta. En este sentido, se observa que el propósito escatológico y las referencias astronómicas contenidas en el escrito que comentamos, no constituyeron un aspecto conflictivo en Lacunza, puesto que tanto la religión como la ciencia fueron compatibles en el pensamiento del sacerdote criollo. Como han señalado los historiadores de la ciencia Peter Bowler e Iwan Morus las relaciones entre la fe y la ciencia no siempre son problemáticas, sino que pueden coexistir o la primera puede inspirar a la segunda para gestar alguna teoría sobre la naturaleza ${ }^{4}$.

En consecuencia, en esta comunicación proponemos que las menciones astronómicas en La Venida del Mesías en gloria y majestad, de Lacunza, dejan de manifiesto que el conocimiento de la astronomía no se observa únicamente entre los exponentes del Chile republicano, como se señala a menudo cuando se da cuenta de la evolución de esta disciplina en Chile, sino que tiene una génesis ya en el Siglo XVIII; uno de cuyos adelantados es justamente Lacunza. Y además, dichas menciones astronómicas que aquí analizaremos, nos dejan en mejor pie para estudiar la relación entre ciertos tópicos de la religión y la ciencia, tales como los enfoques sobre el cosmos, el movimiento de los astros, los niveles del cielo o la función del sol en relación a los planetas, entre otros. Dicha vinculación está claramente presente en el texto anteriormente citado, en especial en el Tomo III de su obra5.

\section{MANUEl LACUNZA Y SU FORMACIÓN INTELECTUAL}

Manuel Lacunza nació en Santiago, el 19 de julio de 1731 en el seno de una familia criolla de prósperos comerciantes; en 1741 ingresó al Convictorio de San Francisco Javier, perteneciente a los jesuitas, lugar

4 P. Bowler - I. Morus, Panorama general de la ciencia moderna (Crítica, Barcelona: 2007) 430.

5 M. Lacunza, La Venida del Mesías en Gloria y Majestad, Tomo III (Impreso por R. Ackermann, Londres, 1826) 287. 
donde aprendió las primeras letras y egresó en 1747 obteniendo el grado de Maestro en Filosofía ${ }^{6}$. A través del estudio de esta última disciplina, el sacerdote ignaciano obtuvo algunas nociones de ciencia, toda vez que, en el siglo XVIII en Chile, tanto la física como la astronomía se enseñaban como partes de la filosofía. Esta última que se impartía en los establecimientos educativos de la Compañía de Jesús, era marcadamente escolástica, privilegiándose la lectura de los textos de Aristóteles más una modesta complementación con algunos conocimientos de ciencias naturales y matemáticas ${ }^{7}$.

El mismo año que se graduó en el Convictorio de San Francisco Javier, Lacunza ingresó también a la Compañía de Jesús, con tan solo dieciséis años. Se ordenó sacerdote en 1755, siendo designado maestro de gramática en el Colegio Máximo ${ }^{8}$. En esta institución Lacunza recibió instrucción en matemática, lógica y física, como parte del plan de estudios que tenía esta corporación para la formación de los sacerdotes ${ }^{9}$. Por lo cual, se observa que el religioso ignaciano tuvo su primer acercamiento con la ciencia mientras estudiaba en el Convictorio de San Francisco Javier y en el Colegio Máximo. Además, Lacunza indagó sobre temas científicos de forma personal, sintiendo gran atracción por la matemática, la geometría y la astronomía; empero, fue una formación limitada debido a la falta de libros especializados, de maestros e instrumentos científicos ${ }^{10}$.

Tras la expulsión de los jesuitas de América en 1767, el religioso chileno permaneció en la ciudad italiana de Ímola, aunque también estuvo por breves períodos en Roma y Venecia ${ }^{11}$. Durante su exilio en Europa,

$6 \quad$ W. Hanisch, "El Padre Manuel Lacunza (1731-1801). Su hogar, su vida y la censura española", Historia 8 (1969), 177-179.

7 Ver W. Hanisch, En torno a la filosofía en Chile (1594-1810) (Ediciones Historia, Santiago, 1963) 38-43.

8 W. Hanisch, "El Padre Manuel Lacunza..., 181-182.

9 A. Contreras, "La enseñanza jesuita en Chile colonial: sus colegios, universidades y una aproximación a sus métodos y contenidos", Revista Historia de la Educación Latinoamericana 16 (2013) 46.

10 W. Hanisch, "El Padre Manuel Lacunza..., 182-183.

11 Hay pocas fuentes para reconstruir la estadía de Lacunza en Italia, unas de ellas son unas breves cartas compiladas tiempo después de su muerte. Para un análisis detallado sobre las misivas del jesuita chileno y su vida en Europa se puede consultar en C. Rolle, "Las cartas de la vida. Manuel Lacunza, el viaje y el exilio", Anales de Literatura Chilena 24 (2015). 
Lacunza llevó una vida solitaria dedicada al estudio de la Biblia y a la escritura de su obra; su inclinación por la lectura la podemos observar en una de sus cartas escritas en Bolonia en 1791, en donde señala que durante su estadía en esta ciudad aprovechó de registrar algunas librerías y a divertir un poco su hipocondría ${ }^{12}$. Asimismo, continuó con su afición a la astronomía y a la contemplación de los astros, tal como lo han destacado algunos autores, entre estos Francisco Mateos quien señala que Lacunza "tenía la costumbre muy singular de acostarse al apuntar el día, o poco antes según las estaciones. Acaso, arrebatado por el gusto de la astronomía que había tenido desde su juventud le era grato estar en vela mientras estaban visibles los astros en el cielo"13.

El estudio científico no fue un aspecto exclusivo de la personalidad de Lacunza, ya que varios jesuitas americanos expulsos continuaron con sus estudios sobre la realidad natural en diversas disciplinas; participando también en controversias con otros naturalistas y sabios europeos sobre la condición peculiar de la naturaleza americana, buscando contestar a los errores, imprecisiones, lugares comunes e interpretaciones de textos de los ilustrados; en especial para replicar a los escritos de Buffon, Raynal, Pauw y Robertson ${ }^{14}$. Así, en este contexto, Lacunza deja de manifiesto su interés científico en La Venida del Mesías en Gloria y Majestad, obra cuyos primeros esbozos los comenzó en 1775 y culminó de redactar con la versión definitiva en $1790^{15}$. El texto fue impreso póstumamente, bajo el seudónimo de un judío converso al catolicismo de nombre Juan Josafat Ben-Ezra, siendo publicado en diversas ediciones ${ }^{16}$.

12 M. Lacunza, "Bolonia y Mayo 15 de 1791", en J. Espejo, "Cartas del padre Manuel Lacunza", Revista Chilena de Historia y Geografia 13 (1914) 217.

13 F. Mateos, "El Padre Manuel Lacunza y el Milenarismo", Revista Chilena de Historia y Geografía 115 (1950) 142.

14 N. Guasti, "Los jesuitas españoles expulsos ante la disputa del Nuevo Mundo", en A. De Francesco, L. Mascieli y R. Nocera (coord.), Entre Mediterráneo y Atlántico: Circulaciones, conexiones y miradas, 1756-1867 (Fondo de Cultura Económica, Santiago, 2014) 103.

15 W. Hanisch, "El Padre Manuel Lacunza, 207.

16 En este artículo no abordaremos las ediciones sobre La Venida del Mesías en gloria y majestad, ya que nuestro interés está abocado a un análisis de la astronomía presente en su obra. Para profundizar en las diversas versiones sobre este escrito véase en A. De Zaballa, "La Venida del Mesías de Manuel Lacunza: primeras ediciones y crítica", Anuario de Historia de la Iglesia 11 (2002). 
En esta obra, su autor aparte de incluir algunas referencias científicas, empleó en su metodología descriptiva un manifiesto influjo del método utilizado por los naturalistas en el siglo XVIII; al respecto Frédéric Martínez ha expresado que "la influencia del método experimental es muy clara en Lacunza. Define la Biblia como un objeto de estudio científico, lo compara con el cosmos y se asimila así mismo como un astrónomo" ${ }^{17}$. En consecuencia, la práctica de la astronomía no solo fue un aspecto que el jesuita chileno efectuó de manera observacional, sino que también le posibilitó estudiar la Biblia y generar sus planteamientos escatológicos desde la racionalidad y con el método que esta ciencia le proporcionaba.

Además, en La Venida del Mesías en Gloria y Majestad se evidencian algunos elementos racionalistas en el pensamiento teológico de Lacunza provenientes de la Ilustración Católica; uno de ellos corresponde a lo que el historiador Mario Góngora ha definido como un "utopismo" de corte racionalista, el que se puede observar en algunas referencias científicas aparecidas en la obra del jesuita chileno y que conformaron una imagen quimérica sobre los acontecimientos que ocurrirían tras la segunda venida de Cristo ${ }^{18}$. Finalmente, la muerte lo sorprendió el 18 de junio de 1801 en Ímola, Italia.

\section{La Ciencia en la época de Lacunza en Chile}

Chile en el siglo XVIII, si bien presentaba diversas manifestaciones científicas y tecnológicas, no poseía una tradición y un interés radicalizado por tales expresiones; así la idea de ciencia para este período puede identificarse con la filosofía, con los contenidos entregados en la enseñanza de las universidades y casas de estudio vinculados a las distintas órdenes religiosas asentadas en el país, y con la elaboración de algunos mapas regionales ${ }^{19}$. Así como también, con algunos discursos con

17 F. Martínez, "Milenarismo y defensa de la fe en el siglo de las luces: la obra del jesuita chileno Manuel Lacunza", Revista Historia Crítica 3 (1990) 54.

18 M. Góngora, "Aspectos de la Ilustración Católica en el pensamiento y vida eclesiástica chilena (1770-1814), Historia 8 (1969) 60.

19 La construcción de la cartografía se efectuaba en base a la información dada por los indígenas y los lugareños, en este sentido, la cartografía jesuítica colonial registró los componentes físicos y sociales del territorio a través de las misiones y viajes de exploración. Ver L. De LasA - M. T. Luiz, "Representación del espacio patagónico. Una interpretación de la cartografía jesuítica de los siglos XVII y XVIII”, Cuadernos de Historia 35 (2011). 
eventuales descripciones acerca de la naturaleza y del cuerpo físico en general del país, aportadas por algunos autores jesuitas, por ejemplo, en sus Cartas Anuas. Y también podemos observar que estas expresiones científicas, se complementan con la presencia de algunos jesuitas alemanes farmacéuticos como Josef Pausch, Johannes Smaldpaner, Johannes Saitor y Josef Zeitler ${ }^{20}$. Este último fue también el primer estudioso de las aguas minerales del país y el poseedor de la más amplia biblioteca médica existente a la fecha en Chile. Además dirigió una de las boticas de los jesuitas por más de veinte años ${ }^{21}$.

También, muchos autores de órdenes religiosas en este período escriben Historias Naturales, que se remontan ya al siglo XVII, en las que describen las características climáticas y mineralógicas, las vicisitudes geográficas, orográficas, potamológicas y las notas más relevantes de los especímenes de la flora y fauna; además, dan cuenta de observaciones sobre el clima y los temblores, observaciones geofísicas sobre las variaciones de la declinación magnética, estudios sobre las mareas, los vientos, las corrientes marinas y las interrelaciones entre los volcanes y los terremotos $^{22}$.

En relación a las Cartas Anuas, nada más a manera de ilustración, recordemos que los jesuitas siempre daban cuenta de las características geográficas y climatológicas de los lugares que visitaban; así, por ejemplo, en relación a la Isla de Chiloé y de la región austral en general, hacen descripciones en estos términos:

"Está la Isla de Chiloé en largo desde $44^{\circ}$ hasta $46^{\circ}$ de altura del sur, tiene de ancho por donde mas solo siete leguas, es muy fragosa y estéril por estar toda ocupada de una montaña tan cerrada que por mas que la rocen, al segundo año, por las muchas lluvias, no da lugar a sementeras.

20 A. Krebs - U. Tapia - P. Schmid, Los Alemanes y la Comunidad chileno-alemana en la Historia de Chile (Liga Chileno-Alemana, Santiago, 2001) 18.

21 A. Krebs - U. TAPIA - P. SCHMid, Los Alemanes y la Comunidad chileno-alemana..., 18.

22 Sobre este último fenómeno, los jesuitas llevaron registro sobre los terremotos y sismos que ocurrieron en el Reino de Chile, describiendo las actitudes que tuvieron los chilenos ante este tipo de manifestaciones de la naturaleza. Un estudio sobre este tipo de registro se puede encontrar en J. VALEnZuela, "Relaciones jesuitas del terremoto de 1730: Santiago, Valparaíso y Concepción”, Cuadernos de Historia 37 (2012). 
En medio de la longura de esta isla, está la pequeña ciudad de castro donde reciden 7 u 8 hermanos nuestros" ${ }^{23}$.

Lo anterior, ilustra el énfasis por describir la naturaleza autóctona de los lugares que visitaban y que caracterizaba a los sacerdotes de esta Orden, y si bien, la ubicación geográfica de Chiloé está errónea pues hoy sabemos que la isla está ubicada entre los $41^{\circ} 46^{\prime}$ y $43^{\circ} 26^{\prime}$ de Lat. y a $73^{\circ} 20^{\prime}$ y $74^{\circ} 23^{\prime}$ de Long. ${ }^{24}$, de todos modos es un loable esfuerzo de determinación de coordenadas geográficas para la época en que se escriben estas notas (1618-1619).

Y en relación a las Historias Naturales, recordemos los casos de: Alonso de Ovalle (1603-1651), que nos ha legado su Histórica Relación del reino de Chile y de Diego de Rosales (1601-1677) que nos ha dejado su Historia General del reino de Chile, Flandes Indiano. Es frecuente también encontrar en estas obras descripciones sobre los nativos y lugareños; es el caso de los jesuitas Felipe Gómez de Vidaurre (1748-1818) quien en su manuscrito Historia Geográfica Natural y Civil del Reino de Chile, (1789), da cuenta de las comidas, ceremonias, juegos, formas de gobierno y costumbres de los nativos chilenos ${ }^{25}$. Y lo propio hace Ignacio Molina (1740-1827) que de manera más lata da cuenta de los usos y las costumbres de los araucanos, y en general de sus principales actividades sociales, en su obra: Compendio de la Historia Geográfica, Natural y Civil de Chile; esta última escrita en Bolonia. Tales referencias nos indican que Lacunza perteneció a una orden religiosa que contribuyó al estudio científico de la Capitanía, la cual combinó las actividades misionales y educativas con la investigación científica ${ }^{26}$.

Y en cuanto a las expresiones tecnológicas de la época, estas están asociadas a la arquitectura, a la construcción de fuertes, a la construcción de naves en los astilleros de la ribera del Maule, de Valdivia o Chiloé, a la erección de hospitales de caridad, fundición de campanas, construcción

23 Cartas Anuas de la Provincia de Paraguay, Chile y Tucumán de la Compañia de Jesús, Documentos para la Historia Argentina, Tomo XX (Universidad de Buenos Aires, Facultad de Filosofía y Letras, Buenos Aires, 1929) p.12.

24 F.S. Astabu-Ruaga, Diccionario Geográfico de la República de Chile (2da Edición s/ indicación de imprenta, Santiago, 1899) 231.

25 Ver. Vidaurre, Felipe Gómez de: Historia Geográfica Natural y Civil del Reino de Chile (Imprenta Ercilla, Santiago, 1889).

26 Ver. A. Prieto, Missionary scientits: Jesuit Science in Spanish South America, 1570 1810 (Vanderblit University Press, Nashville, 2011). 
de canales y otras obras públicas, las que estuvieron relacionadas a las políticas borbónicas de construcción de ciudades y pueblos, en la cual tuvieron una relevancia los ingenieros militares. Así, por ejemplo en relación a los astilleros, recuérdese que la industria naviera del siglo XVIII en Chile tuvo un impulso a partir de 1748, con la llegada de un grupo de artesanos especialistas alemanes que le insuflaron actividad al astillero de la ribera del Maule ${ }^{27}$; lo que se sumaba a los astilleros de Valdivia y Chiloé, "donde se construía la mayoría de los barcos que hacían el comercio entre Perú y Chile"28. En cuanto a la edificación de hospitales de caridad que motivaron esfuerzos técnicos e ingenieriles en este período, recordemos a manera de ilustración, la construcción del Hospital San Francisco de Borja en $1786^{29}$, en Santiago, o el Hospital San Juan de Dios reedificado en 1791, bajo la dirección de Toesca, también en Santiago ${ }^{30}$. Y en relación a la construcción de canales, recuérdese el trazado y construcción del canal de Maipo, dirigido por el jesuita Johann Bitterich $^{31}$. Y en relación a la construcción de ciudades recuérdese por ejemplo, la de San Felipe (1740), Cauquenes, Talca y San Fernando (1742), Copiapó $(1744)^{32}$, la villa de Alhué $(1753)^{33}$ o Casablanca $(1755)^{34}$, o la refundación de Illapel $(1790)^{35}$, entre otras.

En este contexto, las ciencias exactas que cultivó Lacunza en Chile y que estuvieron presentes en su obra teológica, se desarrollaron de manera muy precaria y contó con muy pocos exponentes, encontrándose atrasada en relación a los descubrimientos y avances que se venían gestando en Europa. En cuanto a las matemáticas, estas se emplearon en las mensuras de tierra, en las anotaciones contables de la Real Hacienda y

27 Ver M. PInO, Nuestra Cultura Tecnológica. Desde sus orígenes hasta fines del Siglo XIX (Universidad de Santiago, Stgo., 2003) 253.

28 G. Lohmann, Historia Maritima del Perú. Siglos XVII y XVIII, T. IV (Instituto de Estudios Histórico-Marítimos del Perú, Lima, 1975) 284.

29 E. Laval, Historia del hospital San Juan de Dios de Santiago (Asociación Chilena de Asistencia Social, Santiago, 1949) 71.

30 E. Laval, Historia del hospital..., 82.

31 A. Krebs, U. Tapia y P. Schmid, Los Alemanes y la Comunidad chileno-alemana, 16.

32 Ver S. Lorenzo, Origen de las Ciudades Chilenas. Las fundaciones del Siglo XVIII (Ediciones Universitarias de Valparaíso, Pontificia Universidad Católica de Valparaíso, Valparaíso, 2013) 21.

33 S. Lorenzo, Origen de las Ciudades Chilenas..., 164.

34 S. Lorenzo, Origen de las Ciudades Chilenas..., 141.

35 S. Lorenzo, Origen de las Ciudades Chilenas..., 105. 
se empleó la regla de tres para la burocracia civil y eclesiástica. Además, las obras públicas y arquitectónicas utilizaron el cálculo y la geometría, estando estas en manos de los ingenieros militares quienes tuvieron formación en este tipo de ciencias. Asimismo, la matemática se enseñaba en las escuelas elementales a través de las tablas de memorización, las que contenían las cuentas u operaciones básicas ${ }^{36}$.

A nivel universitario la matemática se enseñó en la Real Universidad de San Felipe fundada en 1747, aunque sus funciones académicas se iniciaron recién en 1758. La cátedra de esta disciplina estuvo a cargo del fray dominico Ignacio de León y Garavito, quien fue un matemático autodidacta y que estaba acreditado únicamente por sus trabajos cartográficos. Los estudiantes fueron escasos, ya que la cátedra no dispensaba grados; sin embargo, esta mantuvo cierta regularidad hasta el fallecimiento de su profesor en 1767. Los contenidos curriculares incluían Los Elementos de Euclides, nociones de geografía y cosmografía ${ }^{37}$.

La física y la astronomía, por su parte, fueron universos cognitivos subsumidos en las cátedras de filosofía, en las que se enseñaba la metodología aristotélica bajo una fuerte influencia escolástica ${ }^{38}$. La física no se enseñó a nivel experimental en los establecimientos educacionales, dada la falta de gabinetes e instrumental adecuado para realizar este tipo de actividades. La astronomía también careció de instrumentos para efectuar observaciones celestes a nivel científico y no se establecieron observatorios. No obstante, algunas expediciones científicas realizaron observaciones físicas y astronómicas, aunque estas no dejaron ningún tipo de institución o tradición científica a su paso en el Reino de Chile. Entre ellas recordemos las exploraciones de los viajeros franceses Louis Feuillée (1660-1732) y Amadée Frezier (1682-1773), quienes arribaron a la Capitanía entre los años 1709 y 1712-1713 respectivamente ${ }^{39}$ y dieron una somera visión de ciertos especímenes propios del cuerpo físico del país en obras tales como: Journal des observations physiques, mathématiques et botaniques, Faites par l'ordre du Roy sur les Côtes Orientales

36 R. Hernández, "Chile conquista su identidad con el progreso. La enseñanza de las matemáticas, 1758-1852”, Historia 23 (1988) 137.

37 R. Hernández, "Chile conquista su identidad..., 138.

38 A. Dougnac, "Panorama de la ciencia en Chile en el siglo XVIII", Cuadernos de la Universidad de Chile 2 (1983) 59-62.

39 D. Barros Arana, Historia General de Chile. Tomo V (Centro de Investigaciones Diego Barros Arana, Santiago, 1999) 381-385. 
de l'Amérique Méridionale, et dans les Indes Occidentales, depuis l'année 1707, jusques en 1712, (de Feuillée) o en Relation du voyage de la mer du Sud, aux côtes du Chili, du Pérou et de Brésil, fait pendant les années 1712, 1713, et 1714. (de Frezier).

Los libros científicos que circularon en el Chile colonial y que pudieron estar a disposición de Lacunza, antes de la expulsión de los jesuitas, fueron escasos durante el siglo XVII y se trataban de versiones antiguas y que no contemplaban los últimos descubrimientos de la época; entre ellos cabe mencionar: Euclides matemático, Geometría, de Friso, y Física de Prado $^{40}$. Para el siglo XVIII hubo un aumento de la circulación de libros como símbolo de la cultura letrada y de la Ilustración, estando los textos de ciencia en manos de obispos, gobernadores y profesores. Por ejemplo, la biblioteca del obispo de Santiago, Manuel de Alday (1712-1789) incluía algunos tratados de astronomía, matemática y geometría ${ }^{41}$. La importancia de Alday, para los términos de este artículo, consiste en que bajo su gobierno eclesiástico ocurrió la expulsión de los jesuitas. Alday estuvo en posesión de una de las bibliotecas más voluminosas y tuvo una cercanía a Lacunza y su familia. ¿Habrá tenido acceso el jesuita chileno a alguno de los textos científicos perteneciente a la colección de Alday? Desconocemos si ocurrió esto, pero sí quedó de manifiesto que Lacunza lamentó el fallecimiento del obispo de Santiago, en una carta fechada el 9 de octubre de 1788, en Ímola. En ella acotó: "Nos ha sido infinitamente sensible la muerte de nuestro señor Obispo Alday, como que era nuestro verdadero padre que nos amaba con verdad" 42 .

Cabe mencionar que el desarrollo científico en Chile, en la época que vivió Lacunza, fue marginal en relación a otros reinos de la América española, pues aquí comenzaron a asimilar la ciencia moderna de forma tardía, tan solo en las últimas tres décadas del siglo XVIII ${ }^{43}$. En consecuencia, el religioso ignaciano vivió en un período en que la ciencia tuvo muy pocas manifestaciones culturales, aunque lo aprendido en su

40 I. CRuz, "La cultura escrita en Chile (1650-1820). Libros y bibliotecas", Historia 24 (1989) 127.

41 I. Cruz, "La cultura escrita en Chile..., 146.

42 M. Lacunza, "Ímola y octubre 9 de 1788", en J. Espejo, "Cartas del padre Manuel Lacunza”, 214.

43 J. J. Saldaña, "Ilustración, ciencia y técnica en América", en D. Soto, M.A. PuigSamper y L.C. Arboleda, La Ilustración en América Colonial (CESIC, Madrid, 1995) 34 . 
estancia en el país le permitió plasmar su interés por el conocimiento de la naturaleza, en su obra teológica.

\section{Los CONOCIMIENTOS ASTRONÓMICOS DE LACUNZA}

$\mathrm{Al}$ parecer, el interés de Lacunza por la astronomía vendría ya de sus años mozos y asociado a su hábito de acostarse al amanecer ${ }^{44}$, pues a las altas horas de la noche le permitían observar mejor los astros cuando los cielos estaban despejados, lo que era su mayor pasión. La astronomía, por tanto, formaba parte de su bagaje intelectual, y se desarrolló mientras permaneció en el país y, por consiguiente, representa una parte importante de los contenidos que recibían y/o enseñaban los jesuitas mientras residían en América.

De acuerdo con el historiador Mario Góngora, el jesuita chileno se instruyó en astronomía gracias a la lectura del texto del abate francés, Nöel-Antoine Pluche (1688-1761): Espectáculo de la Naturaleza y la Historia del Cielo, la cual fue muy difundida en España, Italia y América ${ }^{45}$. Y no cabe duda que Lacunza leyó la obra de Pluche, pues justamente nuestro jesuita cita a este autor en la sección: "El Cielo Nuevo y Tierra Nueva”, de su obra, en estos términos:

“¿Pues no había antes del diluvio estas cuatro estaciones? No, amigo no las había, según yo pienso y según han pensado antes de mí algunos otros autores graves, religiosos y píos (véase entre otros al religiosísimo y elegante autor del espectáculo de la naturaleza, tomo VI, edición de Nápoles, desde la página 255)”“46.

Ahora, es probable que la lectura de este libro la haya realizado Lacunza mientras estuvo en Chile, ya que este se encontraba en las bibliotecas de los establecimientos de la Compañía de Jesús en el país, al igual que otros manuscritos científicos y teológicos que poseía esta congregación ${ }^{47}$. En efecto, recuérdese que Hanisch menciona que los jesuitas en sus bibliotecas, tenían obras científicas escritas por sus propios herma-

44 M. Vega, "Manuel Lacunza", Historia de la Literatura Chilena de la Conquista y de la Colonia, Tomo II (Editorial Nascimento, Santiago, 1980) 102-103.

45 M. Góngora, "Aspectos de la Ilustración Católica..., 62.

46 Lacunza, Manuel: La Venida del Mesías en Gloria y Majestad (Selección, prefacio y notas de Mario Góngora) (Editorial Universitaria, Santiago, 1969) 126.

47 A. De Tesanos, "El isomorfismo de las bibliotecas jesuitas (siglos XVI-XVII), Revista de Historia Social y de las Mentalidades 18 (2014) 132. 
nos; v. gr.: Tratado sobre los ocho libros de la física (1698), Disputas sobre los libros físicos de Aristóteles y sobre Metafisica (2 vol. 1727, 1728), Disputas sobre seis libros de la Física de Aristóteles (1727), Física aristotélica ilustrada con inventos curiosos de autores, de A. Saajosa (s/f), o el Tratado de los Principios y las causas $(\mathrm{s} / \mathrm{f})^{48}$.

Pluche no fue el único autor que puede haber influenciado a Lacunza en el estudio de la astronomía, puesto que las obras del destacado matemático y astrónomo jesuita, Athanasius Kircher (1602-1680), también eran conocidas por los religiosos chilenos pertenecientes a esta orden ${ }^{49}$. Este sabio alemán radicado en Roma, creó la linterna mágica (antecedente de la cinematografía) y publicó su Magneticum naturae regnum (1667) y El Mundo subterráneo; el primero, tal como lo indica el título alude a las distintas propiedades magnéticas observadas en la naturaleza, y el segundo, presenta estudios de espeleología. Kircher publicó obras sobre disciplinas tales como: la astronomía, la geología, la espeleología, la lingüística, la física, el arte y la matemática, entre otras ${ }^{50}$. De acuerdo con José Emilio Burucúa, Kircher contribuyó en los mecanismos exegéticos que emplearon los jesuitas al momento de estudiar las sagradas escrituras y para el caso particular de Lacunza, deben haber influido en su visión científica, la cosmología y la filosofía de la historia del jesuita alemán ${ }^{51}$. En consecuencia, el teólogo Lacunza articuló una formación científica tanto por las enseñanzas recibidas en las corporaciones a las que asistió, cuanto por su manera autodidacta de sus estudios mientras permaneció en Chile, plasmando luego sus inquietudes sobre el devenir de los astros, en la obra que estamos analizando.

48 W. Hanisch, En torno a la Filosofía en Chile..., 54-69.

49 En las Biblioteca Nacional se han encontrado algunos ejemplares de las obras de Kircher, las que pertenecieron a los jesuitas tras su expulsión en 1767. Un detallado análisis de estos manuscritos se puede consultar en C. Acuña (ED.), La curiosidad infinita de Athanasius Kircher: Una lectura a sus libros encontrados en la Biblioteca Nacional de Chile (Ocho Libros, Santiago, 2012).

50 Para una visión panorámica sobre la producción intelectual de Kircher, véase en N. Trujillo, "Los laberintos de la curiosidad: el destino, la vida y el legado de Athanasius Kircher S.J.", en C. ACUÑA (ED.), La curiosidad infinita de Athanasius Kircher..., 11-21.

51 J.E. BurucÚA, "Escritura y experiencia en la cultura jesuita barroca: de Athanasius Kircher a Manuel Lacunza”, en C. ACUÑA (ED.), La curiosidad infinita de Athanasius Kircher..., 55. 
En La Venida del Mesías en Gloria y Majestad, Lacunza dividió los cielos en tres partes: aéreo, etéreo y empíreo. Sobre el primero señaló: "El primero llamais aéreo: esto es, toda la atmósfera que circunda por todas partes nuestro orbe terráqueo". Mientras que, del segundo, indicó: "El segundo que llamais etéreo ¿cual es este? Es, decis, todo el espacio inmenso é indefinido donde habitan y nadan la luna, el sol, los planetas, los cometas, las estrellas sin número". Para el tercero, mencionó: "El tercero superior á todos, es el que llamais cielo empíreo, mas allá del cual no hay cosa alguna"52. Para el jesuita chileno el tercer cielo no tiene apoyo en la física moderna, ya que los últimos conocimientos sobre astronomía demostrarían que este supuesto cielo empíreo sería pura imaginación ${ }^{53}$.

Esta división tripartita de los cielos no fue única del teólogo ignaciano, pues su contemporáneo en Chile, el fray dominico, Sebastián Díaz (1741-1812), quien fue prior de la Recoleta Dominica y miembro del claustro de la Universidad de San Felipe, realizó una segmentación similar. En su tratado cosmológico titulado Noticia general de las cosas del mundo por el orden de su colocación, impreso en Lima en 1783, manifestó que el cosmos se fragmentaba en: cielo empíreo (dedicado a las verdades de la religión), firmamento (exponiendo los resultados de la ciencia moderna en materia de cosmografía) y cielo aéreo (abordando los fenómenos meteorológicos) ${ }^{54}$. Por lo cual, esta división celestial fue conocida en Chile, o por lo menos en una parte de su élite ilustrada.

El sistema cosmológico asumido por Lacunza en sus descripciones celestes fue el copernicano, aunque no aparece una alusión explícita al respecto, pero sí queda tácito en su obra. Por ejemplo, se puede apreciar esta preferencia por el modelo copernicano, en su descripción sobre las estrellas y al criticar el recurso de las excéntricas y los epiciclos del modelo geocéntrico. Así, en cuanto a lo primero acota: "Luego cada estrella es un sistema solar y planetario, así como lo es ciertamente nuestro sol: luego cada estrella tiene muchos cuerpos (mas ó menos), que la circundan, como á centro comun de movimiento" 55 . Y en cuanto al recurso de las excéntricas y los epiciclos, utilizados para justificar el modelo geocén-

52 M. Lacunza, La venida del Mesías en Gloria y Majestad, Tomo III, op.cit., p. 278.

53 F. PARRA, El reino que ha de venir: Historia y esperanza en la obra de Manuel Lacunza (Ediciones Universidad Alberto Hurtado, Santiago, 2011) 203.

54 A. de Ávila Martel, "La Universidad y los estudios superiores en la época de Carlos III", en F. Campos (et al.), Estudios sobre la época de Carlos III en el reino de Chile (Ediciones de la Universidad de Chile, Santiago, 1989) 196.

55 M. LACUNZA, La venida del Mesías, Tomo III, p. 292. 
trico, Lacunza lo critica en el tomo primero de su libro, señalando que las pruebas presentadas por sus defensores no resultan suficientes; así, refriéndose al modelo de Ptolomeo señala: "Veo los esfuerzos inútiles que hacen para darles alguna explicacion: oigo las suposiciones que procuran establecer, todas arbitrarias, inverosímiles é increíbles. Contemplo con admiracion los excéntricos y los epicíclicos, adonde se acogen por último refugio" 56 .

En Chile en el siglo XVIII, los sistemas cosmológicos, como el ptolemaico, tychónico o copernicano, ya eran conocidos. A modo de ejemplo, recuérdese que fray Sebastián Díaz en su cosmografía se había referido a ellos sin pronunciarse a favor de ninguno ${ }^{57}$. Cabe hacer notar que en la América colonial algunas congregaciones religiosas se opusieron a la enseñanza del sistema de Copérnico en las universidades; este fue el caso de los dominicos (a la cual perteneció Díaz) en el Virreinato de Nueva Granada, quienes se manifestaron contrarios a que el sacerdote español, José Celestino Mutis (1732-1808), incluyera en su cátedra el enfoque heliocéntrico debido a que contradecía las escrituras ${ }^{58}$.

La inclinación hacia el copernicanismo por parte de Lacunza, tuvo algunas repercusiones en Chile; en efecto, en una carta que el capellán José Sallusti envió a Tadeo Reyes en Santiago el 1 de julio de 1824, este señala:

"El modus operandi en teología no es el que se estila en las ciencias matemáticas, por ejemplo, en astronomía o en las ciencias físicas y materiales, pero yerra, a ejemplo de los teólogos españoles de su época, cuando agrega que el "decantado sistema copernicano" (al cual socarronamente alude el P. Lacunza), es erróneo y sospechoso de herejía” 59.

56 M. Lacunza, La venida del Mesías en Gloria y Majestad, Tomo I, 55.

57 Ver V. IOmmi - I. URiBe, "Esferas: una aproximación a la cosmología renacentista en el Chile colonial”, Asclepio 66 (2014).

58 Mutis entró en una larga controversias con los monjes dominicos por la supremacía de la enseñanza de la ciencia moderna en las universidades neogranadinas, estas discusiones tuvieron manifestaciones tanto educativas, como científicas y religiosas. Para profundizar en esta temática consúltese en D. Sото, "La enseñanza de los "sistemas del mundo" en las universidades de los jesuitas de Quito y Santafé. Siglo XVIII", Revista Historia de la Educación Latinoamericana 2 (2000).

59 J. SAllusti, "Santiago de Chile, $1^{\circ}$ de Julio de 1824. Su amigo capellán.- José Sallusti.- Al Señor Dr. D. Tadeo Reyes”, en E. VAISSE, El Lacunzismo (Imprenta Universitaria, Santiago, 1917) 48. 
$\mathrm{Al}$ asumir el sistema copernicano, el teólogo ignaciano expuso también algunas notas sobre la pluralidad de los mundos y sobre el espacio abierto, y con ello se abrió a la percepción de un universo infinito, pues tal como lo ha estudiado Alexandre Koyré, el hecho de pasar de la cosmología ptolemaica a la copernicana, significó no solo la adopción del heliocentrismo, sino que también significó aceptar de plano el fin de la concepción griega de un universo cerrado a uno infinito ${ }^{60}$. Ambos aspectos Lacunza los tenía muy claro, y comprendía que la infinidad de los orbes ya era una verdad científica aceptada; al respecto, en un momento de su prosa acota: "Los infinitos ó innumerables cuerpos celestes, así luminosos como opacos, así visibles como invisibles (cuya existencia ya es innegable), pueden bien estár todos, ó muchos habitados de una infinitiva muchedumbre y variedad de especies análogas al hombre"61.

Junto a esto, el jesuita chileno describió algunos cuerpos celestes que conforman el firmamento nocturno, tales como las estrellas y los planetas. Sobre las primeras indicó: "Todos los innumerables cuerpos celestes, que llamámos estrellas, deben ser luminosos por sí mismos, pues en la distancia prodigiosa en que se hallan respecto de nuestro sol, no puede recibir de él tanta luz" ${ }^{62}$. Asimismo, Lacunza estaba consciente que no se podía determinar la distancia a otras estrellas de manera confiable, ya que en el siglo XVIII la astronomía estaba recién descubriendo que las denominadas estrellas fijas, poseían movimientos propios, lo cual fue fundamental para establecer el ángulo paraláctico y así medir las distancias estelares ${ }^{63}$. Al respecto, manifestó: "Si se habla de una distancia geométrica y precisa, confiesan todos sincéramente, que esta es imposible determinarla: no alcanza á tanto la trigonometría, ni el cálculo, pues no habiendo paralaje, no puede haber principio cierto sobre que estribar" ${ }^{\prime 6}$. De esta cita se puede colegir que el sacerdote ignaciano conocía la forma para calcular las distancias a otras estrellas, así como parte de los tecnicismos astronómicos, como la paralaje.

60 Ver A. Koyré, Del mundo cerrado al universo infinito (Siglo XXI Editores, Madrid, 1979).

61 M. LaCunza, La venida del Mesías..., 294.

62 M. Lacunza, La venida del Mesías..., 291-292.

63 J. NorTh, Historia fontana de la astronomía y la cosmología (Fondo de Cultura Económica, México, 2001) 300-302.

64 M. LacunZa, La venida del Mesías..., 292. 
En cuanto a los planetas, Lacunza hizo referencia a los seis orbes que giran alrededor del Sol y sus satélites, mencionando por ejemplo que:

"Este [Sol], alumbra y fomenta cuando menos á 16 globos opacos y frios en sí mismos, como son Mercurio, Venus, nuestra Tierra, Marte, Júpiter y Saturno, y fuera de los seis globos primarios, alumbra tambien y fomenta evidentemente á nuestro satélite, que llamamos Luna, á los cuatro de Júpiter, y á los cinco de Saturno"65.

También, el jesuita chileno aludió a otros cuerpos celestes en estos términos: "Sin entrar en este número los cometas, el Herschel y otros" 66 . Lo interesante de esta cita es que Lacunza nombra el Herschel, el cual corresponde a un descubrimiento que realizó el astrónomo germanobritánico, William Herschel (1738-1822), sobre un nuevo planeta que denominó Urano, avistándolo el 13 de marzo de $1781^{67}$. En consecuencia, el teólogo ignaciano incluyó en su obra algunos conocimientos astronómicos que adquirió después de la expulsión de los jesuitas, mientras permaneció en Italia.

Así, el jesuita chileno no solo se destacó por poseer conocimientos de astronomía a nivel teórico, sino que también los poseyó a nivel observacional, ya que estuvo en posesión de un telescopio, convirtiéndose en lo que actualmente denominaríamos: un astrónomo aficionado. Sobre esto mencionó:

"Yo me acuerdo bien, que en sola la espada de Orion compuesta de tres estrellas que mis paisanos llaman las tres Marías, y en el espacio aparente que estas dejan entre sí, conté una vez 42, y esto usando de un telescopio apenas digno de este nombre: pues su vidrio objético no llegará á ocho pies de foco"68.

Lacunza no deja claro si el telescopio lo utilizó en Chile o en Italia, lo que sabemos es que en la Capitanía existieron muy pocos de estos instrumentos, uno de ellos le perteneció al comerciante Manuel Pérez Cotapos, quien facilitó un anteojo de Dollond de 9 pies de largo y sin pie a los científicos españoles José Espinoza y Tello y Felipe Bauzá, miembros

65 M. Lacunza, La venida del Mesías..., 293.

66 M. Lacunza, La venida del Mesías..., 293.

67 J. NorTH, Historia fontana de la astronomía..., 304.

68 M. LaCunZA, La venida del Mesías..., 291. 
de la Expedición Malaspina (1789-1794), quienes emplearon este objeto para determinar la velocidad del sonido en el valle del Maipo ${ }^{69}$.

Otro testimonio sobre telescopios en el período colonial es el que entrega Arturo Aldunate, el cual sostiene que los frailes fundadores de la Universidad de San Felipe, realizaron observaciones a la Luna con un pequeño telescopio traído desde Europa, causando asombro y curiosidad entre las personas ${ }^{70}$. Sin embargo, Aldunate no entrega ningún tipo de documentación sobre esta noticia, quedando tan solo como una anécdota.

Volviendo a Lacunza, sabemos que no solo dirigió su telescopio a la constelación de Orión, sino que también observó las Híades y las Pléyades en la constelación de Tauro, tal como lo señala en un momento de su prosa, en estos términos: "Casi otro tanto me sucedió con las Hiadas y Pleyadas, y generalmente en cualquier parte del cielo ácia [sic] donde enderezaba mi pequeño instrumento" 71 . El teólogo ignaciano no fue el único jesuita que en el exilio hizo referencia a la observación de los cielos, pues su connacional, el abate Juan Ignacio Molina, había destacado la claridad con que se podían observar los cuerpos celestes en Chile, al acotar que: "En efecto, el cielo, despejado casi todo el año de nubes y vapores, mantiene su color azul y permite a las estrellas brillar con todo su esplendor natural"72.

En consecuencia, Lacunza gracias a su obra que estamos comentando, deja claro que poseía un gran bagaje sobre los conceptos, funciones y aplicaciones propias de la astronomía, siendo frecuente las menciones a esta ciencia en su tratado teológico, en especial en el tercer tomo, como ya hemos mencionado. Todo lo cual, nos permite comprender la inclinación hacia los estudios científicos que tuvo el sacerdote ignaciano.

69 T. Donoso, "Práctica científica en América meridional. Experiencias sobre la velocidad del sonido en Santiago de Chile a fines del siglo XVIII", Revista de Geografia Norte Grande 56 (2013) 202.

70 A. Aldunate, Chile mira hacia las estrellas: pequeña historia astronómica (Editora Nacional Gabriela Mistral, Santiago, 1975) 121.

71 M. LacunZa, La venida del Mesías..., 291.

72 J. I. Molina, Ensayo sobre la historia natural de Chile (Ediciones Maule, Santiago, 1986) 25. 


\section{LA ASTRONOMÍA Y LA RELIGIÓN EN LA OBRA DE LACUNZA}

La Venida del Mesías en Gloria y Majestad es un texto íntegramente de teología que buscó dar una explicación a la segunda venida de Cristo en un contexto de convulsiones políticas y sociales. No obstante, la obra de Lacunza posee varias referencias a aspectos científicos como se explicó en los apartados anteriores, lo cual nos permite analizar la relación entre la religión y la ciencia, en particular la correlación entre la información que entrega la biblia sobre la creación de la naturaleza y el conocimiento astronómico de la época. Esto, en un siglo en que las ideas ilustradas exacerbaban el poder de la razón y la ciencia.

En el período en que Lacunza permaneció en Chile la Iglesia estuvo muy unida al poder temporal, siendo el gobernador vicario del monarca quien presidía además las festividades religiosas como símbolo del absolutismo despótico de los borbones. Por consiguiente, el gobernador fue el representante de las políticas ilustradas emanadas desde la Corona con el propósito de modernizar la burocracia ${ }^{73}$. En la América española el proyecto ilustrado de los borbones no fue contrario a la religión, ni tampoco se caracterizó por su excesivo racionalismo, más bien se trató de un movimiento de tipo patriótico que valoró el conocimiento científico como una forma de aprovechar los recursos naturales para el bienestar de los reinos ${ }^{74}$. Asimismo, las congregaciones religiosas fueron las encargadas de impartir educación entre los americanos y de difundir la actividad científica, por lo cual el vínculo entre la religión y la ciencia fue una constante en el siglo XVIII.

Tras la expulsión de la Compañía de Jesús, a Lacunza le tocó vivir en una Europa en que las ideas tanto racionalistas como secularistas avanzaban a través de la difusión de los medios escritos. Un ejemplo de esto fue la Enciclopedia, siendo uno de sus inspiradores el matemático francés, Jean Le Rond D’Alambert (1717-1783), quien manifestó que el conocimiento provenía de los sentidos y no de la revelación bíblica, aunque reconocía formalmente la autoridad de la Iglesia. Así, tanto D'Alambert como Denis Diderot (1713-1784) a través de la Enciclopedia legitimaron La Ilustración al identificar su filosofía con el conocimiento mismo, el que debía validarse por medio de las facultades de

73 J. Valenzuela, Fiesta, rito y política. Del Chile borbónico al republicano (Centro de Investigaciones Diego Barros Arana, Santiago, 2014) 65-66.

74 J. Cañizares-Esguerra, Cómo escribir la historia del Nuevo Mundo. Historiografías, epistemologías e identidades en el mundo Atlántico del siglo XVIII (Fondo de Cultura Económica, México, 2007) 30. 
la mente y la experiencia, oponiéndose a los saberes emanados desde el Estado y la Iglesia ${ }^{75}$. Esta obra propuesta por Diderot y D'Alambert, que queda inserta en el ideario del marco filosófico ilustrado, pretende llevar las luces del conocimiento a todos los espíritus selectos. Es el corazón de una gran empresa que no solo se limita a dar cuenta de las ciencias, sino que además persigue ordenar y clasificar todas las $\operatorname{cosas}^{76}$. De esta forma, el teólogo ignaciano vivió en una Europa en que la razón era la única manera de entender la realidad natural y en una época bullente de revoluciones sociales y políticas, pero en la cual, el elemento escatológico no estuvo ausente ${ }^{77}$.

Como se expresó en un comienzo, la religión y la ciencia no siempre entran en conflictos, pues puede existir un complemento entre ambas. Así por ejemplo, el concepto de leyes naturales proviene de la noción cristiana de Dios como legislador ${ }^{78}$, ante la cual Lacunza se manifiesta muy respetuoso. Por ello, en una carta escrita en Ímola el 7 de diciembre de 1779, indicó: "Solo siendo un bárbaro sin algún respeto a las leyes de la naturaleza podía olvidarme del todo de mi amada madre"79.

La convergencia entre ciencia y religión en La Venida del Mesías en Gloria y Majestad se puede encontrar en varios pasajes, en uno de ellos, por ejemplo, Lacunza se refiere a los cambios que experimentará la Tierra en la segunda venida de Cristo; al respecto señala:

"Esta proposicion bien importante se puede fácilmente probar con el aspecto actual del mismo globo, y con cuantas observaciones han hecho hasta ahora, y hacen cada día los mas curiosos observadores de la naturaleza: mucho mas si este aspecto y estas observaciones se combinan con lo que nos dice la Escritura sagrada" ${ }^{80}$.

Este énfasis convergente entre fe y cuerpo físico, del jesuita chileno, es propia de la Ilustración Católica americana, pues para esta, tanto las revelaciones de la fe y el conocimiento científico fueron parte de la misma empresa tendiente a estudiar la naturaleza como una obra de Dios ${ }^{81}$.

75 R. DARnton, El negocio de la Ilustración. Historia editorial de la Encyclopédie, $1775-$ 1800 (Fondo de Cultura Económica, México, 2006) 6-7.

76 Ver Z. Saldivia, "La Clasificación de las Ciencias", Revista Universum, 24 (2009).

77 F. PARra, El reino que ha de venir..., 34-35.

78 P. Bowler - I. Morus, Panorama general..., 431.

79 M. Lacunza, "Ímola, Diciembre 7 de 1779", en J. Espejo, "Cartas del padre Manuel Lacunza”..., 211.

80 M. LacunZa, La venida del Mesías..., 50.

81 Ver M. Góngora, "Aspectos de la Ilustración Católica..., 43-45. 
Para el teólogo ignaciano, la naturaleza es el ámbito donde se manifiestan los designios del creador, los cuales son estudiados por los científicos; sin embargo, Lacunza, a diferencia de los filósofos naturales de la Ilustración -que eran partidarios de un conocimiento más secularizadola intervención divina era fundamental para entender los mecanismos naturales. Por ejemplo, sobre este punto, al pronunciarse sobre las consecuencias de diluvio universal, manifiesta:

“¿Que causa general fue esta? á mí me parece (en la opinión que sigo) que no fué algun encuentro casual de nuestro globo con algun cometa (como han imaginado posible y aun fácil muchos sábios calculadores de nuestro siglo, como si ya supiesen todos los resortes de la máquina admirable del universo) sino la misma mano omnipotente y sapientísima, aunque invisible, del Criador y Gobernador de toda la máquina" ${ }^{2}$.

De tales palabras dichas por Lacunza, se puede entender que fue partidario de la filosofía mecaniscista, debido a que se refiere a la naturaleza como una máquina gobernada por Dios, ya que los filósofos naturales del siglo XVII, como por ejemplo René Descartes (1596-1650), consideraban que el mundo, así como el universo, era un mecanismo perfecto que poseía leyes inteligibles que podían ser desentrañadas mediante el uso de la razón y del conocimiento matemático. Este tipo de pensamiento influenció tanto a las ciencias de la vida como a las ciencias exactas, tiendo varios cultores entre los siglos XVII y XVIII ${ }^{83}$.

Si bien Lacunza era un seguidor y conocedor de los descubrimientos científicos de su época, tal como se ha mostrado en el apartado anterior, también se mostraba crítico de estos, en particular de algunos filósofos naturales que tenían una postura contraria a Dios y a la creación. En este aspecto, el jesuita chileno menciona:

"Estos filósofos de que hablo, han alcanzado ciertamente grandes luces, y grandes y magníficos conocimientos sobre la naturaleza, ó sobre las obras del Criador; mas en lugar de subir al Criador mismo y parar en él, han parado vergonzosamente en las criaturas, como si estas fuesen el último fin del hombre" ${ }^{84}$.

82 M. Lacunza, La venida del Mesías..., 53.

83 Con respecto a la filosofía mecanicista ver P. DeAR, La revolución de las ciencias. El conocimiento europeo y sus expectativas (1500-1700) (Marcial Pons, Madrid, 2007) 131-160.

84 M. LacunZa, La venida del Mesías..., 296. 
Además, el teólogo ignaciano estaba consciente del límite de la astronomía observacional, ya que esta no podría develar toda la creación de Dios, siendo el intelecto de la divinidad mayor que el del género humano; o en palabras de Lacunza:

"Los mejores telescopios que hasta aora se han podido construir, v. g. de 50, de 100 y aun de 200 pies, nos descubren ciertamente un campo inmenso sobre todo cuanto se habia imaginado. Y no obstante debémos suponer y confesar racional y religiosamente, que estos admirables instrumentos, como obra del ingenio y manos del hombre, no es posible que alcancen á revelarnos todas las obras del altísimo" ${ }^{85}$.

El autor de La Venida del Mesías en Gloria y Majestad escribió en un período en que la astronomía observacional estuvo en auge, debido a que en el siglo XVIII aumentaron los observatorios financiados por los gobiernos, las universidades, las comunidades científicas y las congregaciones religiosas. Además, se creó un mercado de instrumentos astronómicos tanto para fines científicos como para los aficionados, logrando que la ciencia de los astros se propagara a nivel social y que creciera el número de sus profesionales ${ }^{86}$.

Las críticas hacia el conocimiento astronómico que expresó Lacunza no solo fueron hacia los límites que presentaban los telescopios, sino que también el jesuita ignaciano se manifestó crítico frente a los catálogos estelares, puesto que estos no consideraban todas las estrellas, las cuales fueron producto de la creación divina. Por ello, señala que la ciencia solo puede entregarnos una visión parcial de la naturaleza, expresándolo en estos términos:

"Me direis acaso, que ya estas están contadas y puestas en exactísimos catálogos, por los mas diligentes observadores; los cuales apenas han hallado tres mil en ambos hemisferios. Preguntad aora á estos mismos sabios, si realmente no hay mas estrellas que las que se hallan en sus catálogos, y os responderán todos unánimemente, que estas, respecto de las que quedan, no son sino como tres gotas de agua respecto a todo el océano" ${ }^{87}$.

En la astronomía, en la época de la Ilustración, los observatorios europeos hicieron grandes esfuerzos para realizar catálogos estelares lo más preciso posibles, para lo cual se financiaron expediciones que recorrieron

\footnotetext{
85 M. LacunZa, La venida del Mesías..., 291.

86 J. North, Historia fontana de la astronomía..., 289.

87 M. LaCunZA, La venida del Mesías..., 290.
} 
el planeta para llevar a cabo esta tarea, haciendo uso de las últimas innovaciones ópticas, así como en instrumentos de observación ${ }^{88}$.

Otro ámbito en que el jesuita chileno manifiesta sus convicciones frente a la práctica astronómica y la religión es con respecto a la existencia de vida en otros planetas, como se dijo anteriormente, Lacunza es partidario de un universo infinito, compuesto de millones de estrellas y orbes. Sobre esta posibilidad, para el teólogo ignaciano no cabe duda que estas criaturas deberían ser hijos de Dios, señalando: "Que si acaso hay en otros globos otras criaturas análogas al hombre (sean las que fueren y como fueren) todas ellas deben pertenecer á Cristo Jesus, y sujetarse enteramente á su dominacion, pues todas ellas, no menos que nosotros, fueron criadas por él y para él"89.

En consecuencia, para Lacunza el conocimiento científico no contradice la información bíblica o la creación divina, sino que más bien este sería un complemento para desentrañar los misterios de la naturaleza. Por lo cual, los descubrimientos astronómicos de su tiempo también revelaban esta relación, v. gr.: "Todos esto han discurrido estos sabios; cuyo discurso, lejos de oponerse á nuestra creencia divina, ni á la razon natural, antes la sublima, la entiende, la ensalza, y la hace formar un concepto magnífico del Criador de todo" ${ }^{\prime 0}$.

\section{CONCLUSIÓN}

La obra de Lacunza, si bien es esencialmente teológica, se inserta de plano en las preocupaciones astronómicas del Siglo de la Ilustración, pues dicha disciplina estaba en pleno auge en Europa, justamente por esto el autor ignaciano pudo complementar su formación científica en astronomía, pues en Chile si bien había modestas expresiones sobre esta disciplina, no existían aún los medios pedagógicos, ni los textos, ni la difusión adecuada para una formación acabada sobre estos tópicos. Por ello el interés de Lacunza por la astronomía fue el resultado de una motivación personal. Y no es el reflejo del desarrollo científico, ni intelectual del Chile colonial, sino que más bien estas preocupaciones científicas presentes en La Venida del Mesías en Gloria y Majestad son el resultado de un hombre que sintió inclinación por el estudio de la naturaleza y se cultivó lo más posible sobre este tipo de temáticas.

\footnotetext{
J. North, Historia fontana de la astronomía..., 290.

M. LaCunZA, La venida del Mesías..., 294.

M. Lacunza, La venida del Mesías..., 293.
} 
Lo relevante de Lacunza que aun en nuestro tiempo nos llama la atención, es el hecho de haber realizado una asociación cognitiva del saber teológico con el conocimiento científico de la astronomía, con lo cual dejaba de manifiesto que el conocimiento científico no siempre contradice la creación divina, sino que más bien este último sería un complemento más para penetrar en la estructura fenomenológica del universo y explicitar su dinamismo. Lo cual da cuenta que nuestro autor fue conocedor tanto de la tradición religiosa como científica. De la primera, fue un ávido lector de las sagradas escrituras, siendo partidario de su interpretación literal y buscando en ellas tanto refugio intelectual como las condiciones en que se desarrollarían los últimos acontecimientos tras la segunda venida de Cristo. Mientras que, de la segunda, demuestra en el tercer tomo de su obra que estuvo al tanto de los últimos descubrimientos científicos e incluso se manifestó crítico ante la posibilidad de que la ciencia pudiera conocer la realidad natural en su totalidad.

El análisis de La Venida del Mesías en Gloria y Majestad, desde una mirada científica, nos ha permitido vislumbrar que en la historiografía de la ciencia en Chile aún existen algunos vacíos sobre algunos personajes que cultivaron este tipo de conocimiento, debido a que los investigadores han privilegiado el estudio de personas como el abate Molina o las expediciones científicas como manifestaciones de la ciencia en el período colonial, dejando a un lado a otros sujetos que también manifestaron este tipo de preocupaciones. Por lo cual, el estudio de Lacunza nos ha demostrado que en el país en el siglo XVIII existieron personas que tuvieron afición al estudio de la ciencia y que lograron un alto nivel de conocimiento de algunas disciplinas. Para el caso del jesuita chileno, en su obra dejó de manifiesto que poseía entendimiento de algunos conceptos astronómicos, así como de los avances a nivel instrumental de esta disciplina y los progresos en materia observacional y de cálculo que habían logrado los astrónomos en el siglo de la Ilustración. 